\title{
One- and two-stage surgical revision of infected elbow prostheses following total joint replacement: a systematic review
}

\author{
Setor K. Kunutsor ${ }^{1,2^{*}}$ D, Andrew D. Beswick ${ }^{2}$, Michael R. Whitehouse ${ }^{1,2}$ and Ashley W. Blom ${ }^{1,2}$
}

\begin{abstract}
Background: Prosthetic joint infection (PJI) is a challenging complication of total elbow replacement (TER). Potential surgical treatments include one- or two-stage revision; however, the best treatment for elbow PJl is not clearly defined. We conducted a systematic review in accordance with PRISMA guidelines to compare the clinical effectiveness of one- and two-stage revision surgery for elbow PJI using re-infection (recurrent and new infections) rates; mortality; clinical measures of function, pain, and satisfaction; and non-infection related adverse events.

Methods: MEDLINE, Embase, Web of Science, and The Cochrane Library were searched up to June 2019 to identify observational cohort studies and randomised controlled trials (RCTs) that had recruited patients with elbow PJ following TER and treated with one- or two-stage revision. Of 96 retrieved articles, 2 one-stage and 6 two-stage revision studies were eligible. No RCT was identified. Arcsine transformation was used in estimating rates with 95\% confidence intervals (Cls).
\end{abstract}

Results: Staphylococcus aureus was the most common causative organism for PJl of the elbow (24 of 71 elbow PJls). The re-infection rate $(95 \% \mathrm{Cl})$ for one-stage (7 elbows) ranged from $0.0 \%(0.0-79.3)$ to $16.7 \%(3.0-56.4)$ and that for two-stage revision (87 elbows) from $0.0 \%$ (0.0-49.0) to 20.0\% (3.6-62.4). Non-infection related adverse event rate for one-stage (based on a single study) was 16.7\% (3.0-56.4) and that for two-stage ranged from 11.8\% (4.7-26.6) to 20.0\% (3.6-62.4). There were no mortality events recorded following one- or two-stage revision surgery and postoperative clinical measures of function, pain, and satisfaction could not be effectively compared because of limited data.

Conclusions: No strong conclusions can be drawn because of limited data. The one-stage revision may be potentially at least as clinically effective as two-stage revision, but further data is needed. There are clear gaps in the existing literature and studies are urgently warranted to assess the clinical effectiveness of one- and two-stage revision strategies for PJl following TER.

Systematic review registration: PROSPERO 2018: CRD42018118002.

Keywords: Prosthetic joint infection, Elbow replacement, Revision, One-stage, Two-stage, Systematic review

\footnotetext{
* Correspondence: setor.kunutsor@bristol.ac.uk

${ }^{1}$ National Institute for Health Research Bristol Biomedical Research Centre University Hospitals Bristol NHS Foundation Trust and University of Bristol, Bristol, UK

${ }^{2}$ Translational Health Sciences, Bristol Medical School, Musculoskeletal Research Unit, University of Bristol, Learning \& Research Building (Level 1), Southmead Hospital, Bristol BS10 5NB, UK
}

(c) The Author(s). 2019 Open Access This article is distributed under the terms of the Creative Commons Attribution 4.0 International License (http://creativecommons.org/licenses/by/4.0/), which permits unrestricted use, distribution, and reproduction in any medium, provided you give appropriate credit to the original author(s) and the source, provide a link to the Creative Commons license, and indicate if changes were made. The Creative Commons Public Domain Dedication waiver (http://creativecommons.org/publicdomain/zero/1.0/) applies to the data made available in this article, unless otherwise stated. 


\section{Background}

Common complications following total elbow replacement (TER) include implant loosening, periprosthetic fracture, implant failure, triceps insufficiency, nerve palsy, and prosthetic joint infection (PJI) [1]. Compared to lower extremity joint replacements, relatively few elbow replacements are performed. In 2017, as recorded in the National Joint Registry for England, Wales and Northern Ireland and the Isle of Man, approximately 100,000 joint replacements were performed each in knees and hips; whereas only 612 elbow replacements were performed [2]. However, it has been reported that the proportion of complications associated with elbow replacements is greater than that for hip or knee replacements [3].

Prosthetic joint infection (PJI) is a potentially devastating complication of TER and compared with hip or knee replacement, TER is associated with a higher incidence of PJI which affects between 1 to $12 \%$ of patients [4-6]. PJI after TER is associated with significant morbidity [7] as well as increased costs to the healthcare system [8]. Treatment of elbow PJI is a challenging task [9] and the key goals are clearing infection, retaining maximum joint function, and pain relief. Treatment options for elbow PJI include debridement, treatment with antibiotics and retention of the prosthesis (DAIR); resection arthroplasty; and one- or two-stage revision [10]. Resection arthroplasty is generally considered a salvage procedure and used as a last resort in refractory PJI after TER or in patients for whom loss of elbow function is of less concern $[11,12]$. The best treatment for elbow PJI is not clearly defined as choices of treatment strategy are generally based on the treating surgeon's experience and evidence derived from studies of PJI treatment in hip and knee replacement [13]. Based on existing data, the two-stage revision strategy appears to be the most commonly used treatment option for elbow PJI [10]. The one-stage revision strategy may be a putative alternative treatment. An extensive body of evidence suggests that one- and two-stage revision strategies for hip, knee and shoulder PJI are clinically comparable [14-17]; however, the data are sparse and conflicting on the role of these strategies for treating infected elbow prostheses. To our knowledge, no study has yet reviewed the existing evidence by comparing results of published studies that have reported clinical outcomes on any of these two revision strategies. We are also not aware of any randomised controlled trial (RCT) that has compared the clinical effectiveness of the two revision strategies.

To clarify the existing evidence, we conducted a systematic review to compare the clinical effectiveness of the one- and two-stages revision strategies for elbow PJI using infection control as the primary outcome. Secondary objectives included (i) comparing the effectiveness of the two revision strategies based on other clinical outcomes which include mortality; validated measures of function, pain, and satisfaction; as well as non-infection related adverse events and (ii) to explore any gaps in the evidence base.

\section{Methods}

Data sources and search strategy

We registered this review in the PROSPERO prospective register of systematic reviews (CRD42018118002). The review was based on a protocol which was predefined and performed following PRISMA and MOOSE guidelines [18, 19]. We systematically searched MEDLINE, Embase, and The Cochrane library from inception to 25 June 2019 for longitudinal observational studies and RCTs that reported on infection control and/or other clinical endpoints after one- or two-stage surgical revision of an infected elbow prostheses. The computer-based searches employed a combination of free and $\mathrm{MeSH}$ search terms and key words related to the intervention, population and outcomes. Only human studies were searched for, with no restrictions placed on language. The full search strategy is reported in Additional file 1: Table S1. The retrieved citations were initially screened based on their titles and abstracts to assess their potential for inclusion, after which we retrieved potentially eligible articles for full text evaluation. Evaluation of full texts was conducted by two independent authors (S.K.K., A.D.B.) based on the inclusion criteria and any disagreements regarding whether an article should be included or not was discussed, with involvement of a third author (M.R.W) to reach a consensus. We also scanned reference lists of relevant articles (including reviews) for studies missed by the original search. The "cited by" function in Web of Science was used to check for citations of key studies.

\section{Eligibility criteria}

Studies were eligible for inclusion if they (i) were longitudinal observational studies or RCTs that included patients with infected elbow prostheses following TER and were managed by a one- or two-stage revision strategy and (ii) were followed up post-operatively for re-infection (which was defined as recurrence of infection by the same organism(s) and/or re-infection with a new organism(s)) and/or other clinical outcomes such as (a) function [as measured by the Mayo elbow performance score (MEPS); flexionextension range of motion; and triceps function]; (b) pain; (c) satisfaction; or (d) non-infection related complications (such as implant failure, periprosthetic fracture, loosening, haematoma, postoperative instability, nerve entrapment, and triceps insufficiency).

\section{Data extraction and quality assessment}

One author (S.K.K.) initially extracted the data using a data collection form which was standardised for this 
purpose. A second author (A.D.B) checked these data independently with the information in the original articles. Any disagreements were discussed and a third author (M.R.W) was involved to reach a consensus. We extracted the following pieces of information: Author and year of publication, study design, study location, mean or median age at baseline, proportion of male participants, type of revision surgery, revision surgery characteristics, duration of follow-up after revision surgery, number of re-infections after revision surgery, other clinical outcomes, and adverse events. If information about the same study was published twice or more often, we used the most recent publication or the one with most up to date information. The methodological quality of included studies was assessed using the Methodological Index for Non-Randomised Studies (MINORS), a well-established validated instrument designed for assessing the quality of non-randomised studies in surgery [20] and which has been described in previous published papers [14, 15]. Briefly, this tool uses eight pre-defined factors which include: a clearly reported aim, inclusion of consecutive patients, data collected in a prospective manner, endpoints reflecting the aim of the study, study endpoints assessed in an unbiased manner, follow-up duration which is appropriate to the aim of the study, less than $5 \%$ loss to follow-up, and prospective calculation of the study sample size. For each of the domains, the tool assigns a score of 0 for "not reported", 1 for "reported but inadequate", or 2 for "reported and adequate". These are then added into a total score. A score of 16 is regarded as the global ideal score.

\section{Data analysis}

The re-infection rate, which was the primary outcome, was computed from the number of re-infections within the follow-up period following elbow revision surgery divided by the total number of participants with PJI or number of elbow joints with PJI. Re-infection rates with 95\% confidence intervals (CIs) were estimated across the studies by employing the Freeman-Tukey variance stabilising double arcsine transformation [21]. Details of the method have been described previously $[14,15]$. Given the limited data, a pooled analysis was not performed. Non-infection related adverse event rates (computed from the number of adverse events or complications within follow-up period following elbow revision surgery divided by the total number of participants with PJI or number of elbow joints with PJI) with 95\% CIs were also estimated across studies. Stata MP 16 (Stata Corp, College Station, Texas, USA) was employed for all statistical analyses.

\section{Results}

\section{Study identification and selection}

Of 97 records retrieved from the search, we excluded 83 articles based on titles and abstracts. On reviewing the full texts of the remaining 14 articles, we excluded a further 6 articles because (i) intervention was not relevant $(n=5)$ or the article (ii) was a review paper $(n=1)$. This left 8 articles eligible for inclusion in the review (Fig. 1; Table 1) [12, 13, 22-27].

\section{Study characteristics and study quality}

Table 1 provides a summary of baseline characteristics of one- and two-stage revision studies which were eligible. Details on individual study baseline characteristics and methodological quality are reported in Table 2. Of the 8 eligible articles, 6 articles were based on two-stage revision; 1 article evaluated the one-stage revision; and 1 article evaluated both one- and two-stage revision strategies. Overall, there were 9 unique studies comprising 94 elbow joints revised for PJI (92 participants) and 13 re-infections. All included studies retrospectively analysed data based on observational cohort designs or case series. The most common surgical indication for the index TER was rheumatoid arthritis. We did not identify any clinical trials comparing both revision strategies. Studies were carried out in Europe (UK, Germany, and Switzerland) and North America (United States of America). Baseline study level surgery and clinical characteristics could not be compared between the two revision strategies because of the limited number of studies and outcome measures for one-stage revision. Studies reported the diagnosis of PJI in a variety of ways, but was mainly based on the presence of one or more of the following criteria: (i) clinical, haematological and radiological assessments suggesting the diagnosis with persistent swelling and inflammation, high blood indices (such as white cell count, C-reactive protein, or erythrocyte sedimentation rate) and progressive radiolucent lines; (ii) positive results of microbiological culture from preoperative elbow joint aspirate, intraoperative periprosthetic tissue, or sonication fluid of the removed plant; (iii) visible purulence of a preoperative aspirate or intraoperative periprosthetic tissue; (iv) wound findings such as the presence of a sinus tract communicating with the prosthesis; and (v) pathological findings on tissue sections. Staphylococcus aureus (S. aureus) was reported as the most common causative organism for elbow PJI in the majority of eligible studies that provided these data (24 out of 71 elbow PJIs). Intravenous flucloxacillin and rifampin were the most common antibiotics administered following revision surgery. The methodological quality scores of studies included in the review ranged from 9 to 11 .

\section{Revision strategy and re-infection}

Two studies were reported to have evaluated the one-stage revision strategy and comprised of 7 elbow joints revised for PJI (6 participants) and 1 re-infection (Tables 1 and 2). The re-infection rate ranged from $0.0 \%$ (95\% C: $0.0-79.3$ ) 


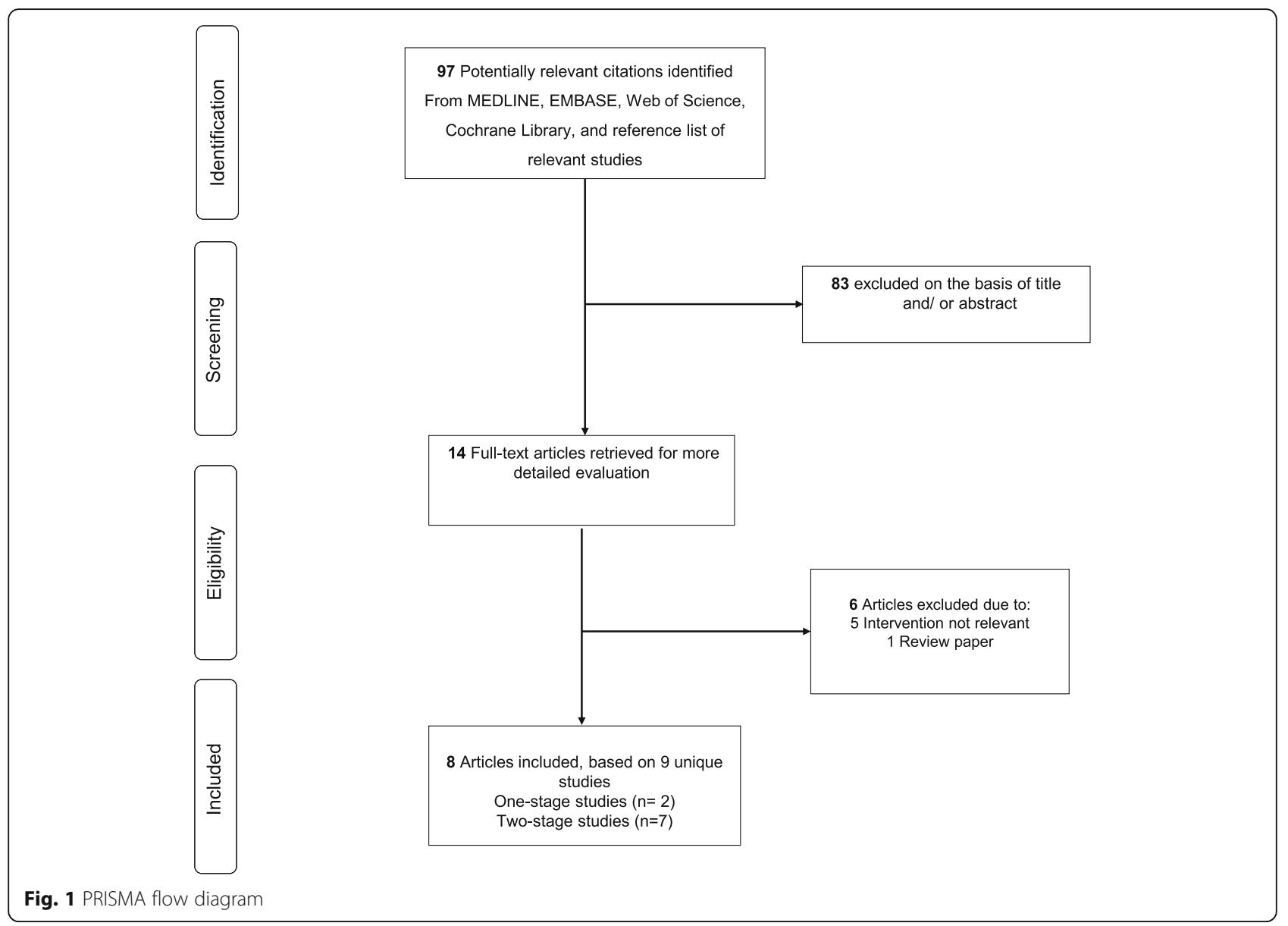

to $16.7 \%$ (95\%CI: $3.0-56.4)$ over a weighted mean followup period of 6.4 years (Fig. 2).

Seven studies comprising of 87 elbow joints revised for PJI (86 participants), reported 12 re-infections following two-stage surgical revision (Tables 1 and 2). The reinfection rate ranged from $0.0 \%$ (95\% CI: 0.0-49.0) to 20.0\% (95\% CI: 3.6-62.4) over a weighted mean followup period of 3.7 years (Fig. 2).

\section{Other post-operative clinical outcomes}

Of the six studies (1 one-stage and 5 two-stage studies) that reported on mortality outcomes, none reported any mortality event associated with the revision strategy (Table 2). The same studies reported on non-infection related adverse events following revision surgery and these included explantation; revision; triceps weakness, insufficiency, or rupture; ulnar fracture; ulnar nerve neuropraxia; skin breakdown; humeral component loosening; and non-union (Table 2). The non-infection related adverse event rate for one-stage which was based on one study was $16.7 \%$ (95\% CI: $3.0-56.4$ ) and that for twostage revision ranged from $11.8 \%$ (95\% CI: 4.7-26.6) to 20.0\% (95\% CI: 3.6-62.4) over a weighted mean followup period of 3.7 years (Additional file 1: Figure S1).
Measures of function and pain between both revision strategies could not be compared using statistical tests because data from one-stage revision was based on limited data points (Table 3). However, median computed values for range of motion, flexion, and MEPS seemed to be better in the two-stage revision group compared with the one-stage group; whereas extension was better in one-stage revision. In 2 two-stage studies that reported on measures of satisfaction, 4 out of 4 patients reported satisfaction with the outcomes in one study and in the other, 6 out of 7 patients were satisfied with their outcomes. One one-stage study reported on this outcome and indicated 4 out of 6 patients as being satisfied with the outcomes post-surgery.

\section{Discussion}

In this literature-based systematic review, the data suggests that one-stage revision may be associated with lower re-infection and non-infection related adverse event rates compared with the two-stage strategy, although the 95\% confidence intervals overlapped and the estimated rates were based on very limited data. Measures of function, pain, and satisfaction could not be compared effectively because of limited data. However, 
Table 1 Summary characteristics of included studies

\begin{tabular}{|c|c|c|}
\hline & One-stage revision & Two-stage revision \\
\hline \multicolumn{3}{|l|}{ Eligible studies } \\
\hline Total number of studies included & 2 & 7 \\
\hline \multicolumn{3}{|l|}{ Participants } \\
\hline Total number of participants & 7 & 87 \\
\hline Total number of re-infections & 1 & 11 \\
\hline Median (IQR) age (years) & $64.9(62.7-67.0)$ & $64.7(59.7-65.0)$ \\
\hline Median (IQR) males (\%) & $0.0(0.0-0.0)$ & $31.0(29.5-35.8)$ \\
\hline \multicolumn{3}{|l|}{ Location } \\
\hline Europe & $2(7)$ & $4(59)$ \\
\hline North America & - & $3(28)$ \\
\hline Asia & - & - \\
\hline \multicolumn{3}{|l|}{ Study and surgery characteristics } \\
\hline Median (IQR) time from index surgery to infection diagnosis (months) & $40.5(40.5-40.5)$ & $38.0(24.0-51.9)$ \\
\hline Median (IQR) duration of infection symptoms (days) & - & $60.4(60.4-60.4)$ \\
\hline Median (IQR) time from index surgery to revision surgery (months) & - & $57.1(48.2-66.0)$ \\
\hline Median (IQR) from infection diagnosis to revision surgery (months) & - & $15.0(15.0-15.0)$ \\
\hline Median (IQR) interval between stages (months) & NA & $6.1(4.7-7.7)$ \\
\hline Median (IQR) follow-up (years) & $5.4(4.0-6.8)$ & $4.1(3.0-4.3)$ \\
\hline Median (IQR) duration of antibiotics (days) & - & $30.5(11.3-49.7)$ \\
\hline Median (IQR) duration of IV antibiotics (days) & $37.2(37.2-37.2)$ & $8.8(8.8-8.8)$ \\
\hline Median (IQR) duration of oral antibiotics (days) & - & $2.5(2.5-2.5)$ \\
\hline Methodological quality (IQR) & $10.5(10.0-11.0)$ & $10.0(10.0-11.0)$ \\
\hline \multicolumn{3}{|l|}{ Baseline clinical characteristics } \\
\hline Median (IQR) Range of motion $\left(^{\circ}\right)$ & - & $72.0(50.0-94.0)$ \\
\hline Median (IQR) Extension & - & $36.3(36.3-36.3)$ \\
\hline Median (IQR) Flexion & - & $101.3(101.3-101.3)$ \\
\hline Median (IQR) MEPS & - & $28.0(22.9-52.5)$ \\
\hline Median (IQR) Pain score & - & $15.0(15.0-15.0)$ \\
\hline
\end{tabular}

IQR Interquartile range, IV Intravenous, MEPS Mayo elbow performance score, NA Not applicable; values are number of studies (number of participants) unless stated otherwise

the data suggested that range of motion, flexion, and MEPS were improved in the two-stage revision group compared with the one-stage group. These findings reflect evidence observed in other joints showing that two-stage revision may potentially be associated with improvement in function, but lower rates of infection eradication compared with one-stage revision $[15,16]$. The findings cannot be compared with previous work, given this is the first ever systematic review to compare the clinical effectiveness of one- and two-stage revision strategies for the management of infected elbow prosthesis.

Based on the limited data, it is difficult to make any conclusions on which revision strategy is more clinically effective. However, it appears the one-stage revision may be potentially at least as effective compared with the two-stage revision strategy, given the low re-infection and adverse event rates. This review has also identified large gaps in the existent literature - it appears that though TERs are associated with higher incidence of PJIs compared with hip or knee replacements [4-6], published series on the use of the two most established PJI treatment strategies are non-existent for elbows. It is obvious that the paucity of data on treatment of elbow PJI reflects the lower incidence of TER utilization [27] compared to hip and knee replacements. This also raises the question on whether infections in elbow arthroplasty run a completely different course clinically and hence can't be treated with one- or two-stage revision surgery in the same way as other joint replacements? As a result of the thin soft tissue envelope of the elbow joint, it is particularly susceptible to infection and this can be worsened by inflammatory conditions (eg, arthritis), 


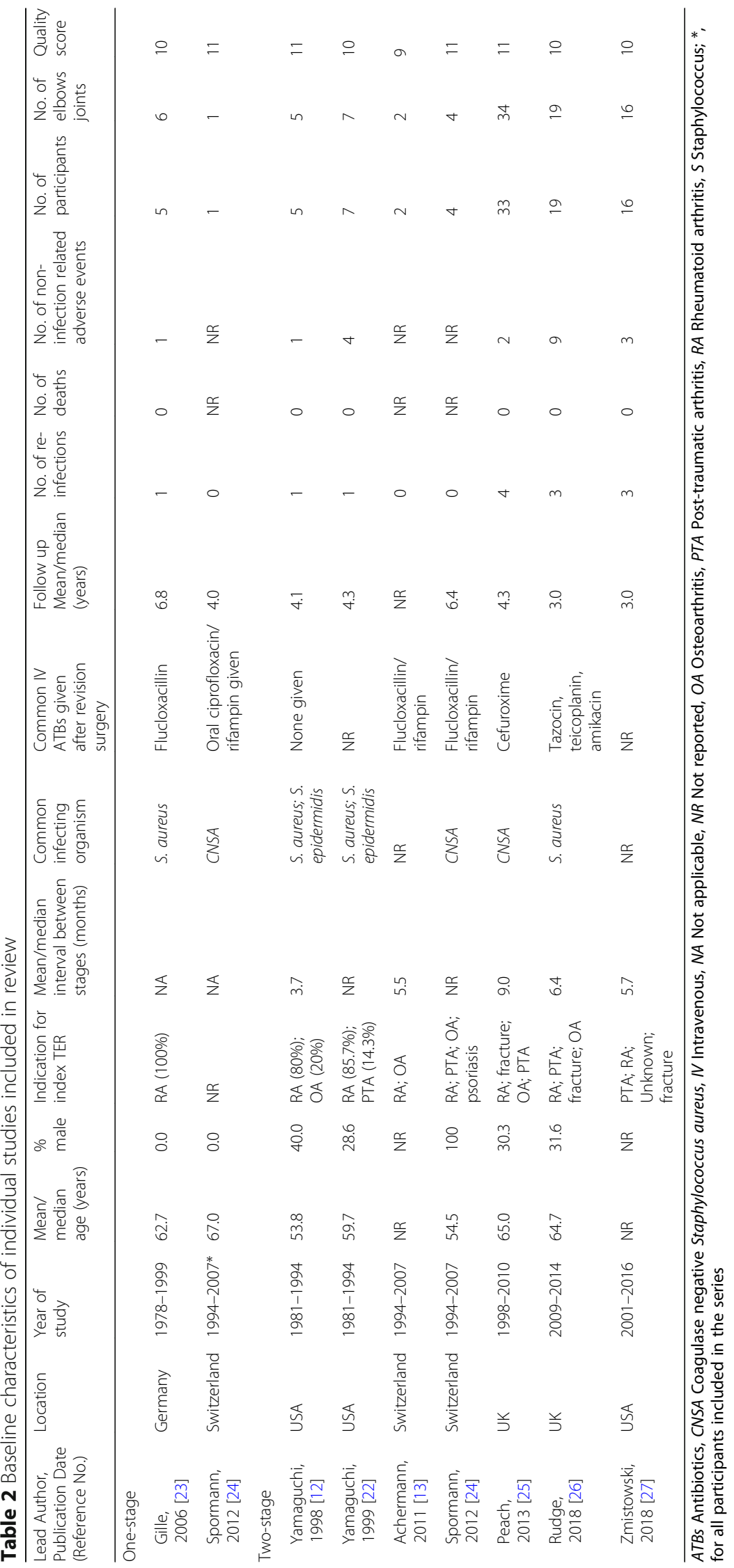




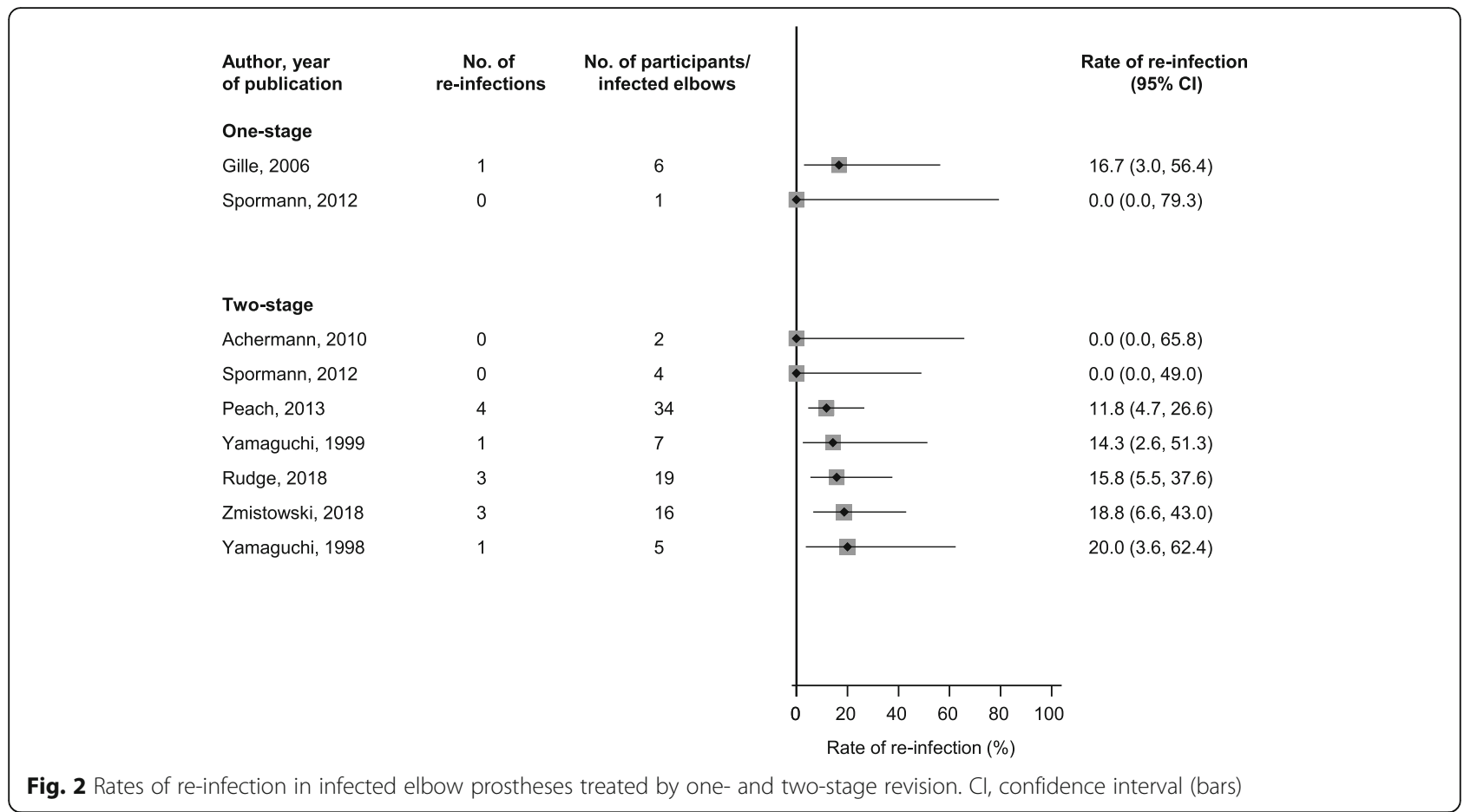

medications used for treating these inflammatory diseases and trauma [1]. Majority of elbow infections are caused by skin bacteria such as S. aureus and S. epidermidis. In contrast to PJIs of the hip and knee joints, prosthetic elbow infections rarely involve systemic symptoms such as fever or malaise [4, 9]. Furthermore, unlike tests for diagnosing prosthetic hip and knee infections, the diagnostic utility of blood testing and joint aspiration for PJI of the elbow is not well established [10]. In a review of the diagnosis and management of prosthetic elbow infection, Somerson and colleagues note that given the lack of objective criteria to diagnose PJI of the elbow, a high index of clinical suspicion is required in addition to knowledge of risk factors and discriminating interpretation of laboratory tests [10]. This especially makes the diagnosis and treatment a challenge. We noted that the average interval between stages in two-stage revision of infected elbow joints was longer (6.1 months) compared to other joints

Table 3 Post-operative clinical outcomes following one- and two-stage revision strategies

\begin{tabular}{lll}
\hline & One-stage revision & Two-stage revision \\
\hline $\begin{array}{l}\text { Median (IQR) Range of } \\
\left.\text { motion ( }{ }^{\circ}\right)\end{array}$ & $90.0(80-90)$ & $104.2(97.0-111.4)$ \\
Median (IQR) Extension & $35.0(20.0-40.0)$ & $18.8(18.8-18.8)$ \\
Median (IQR) Flexion & $120.0(100.0-130.0)$ & $129.6(128.0-131.3)$ \\
Median (IQR) MEPS & $67.6(50.0-80.0)$ & $83.4(77.1-90.4)$ \\
Median (IQR) Pain score & - & $38.6(38.6-38.6)$ \\
\hline
\end{tabular}

$I Q R$ Interquartile range, MEPS Mayo elbow performance score
(3-4 months) $[15,16]$; indeed, except for one study, the majority of studies reported an average of more than 5 months duration between stages. This observation may reflect a more challenging and protracted course of treatment for elbow PJI compared to that of other joints. Although it is clear from the evidence that two-stage revision is currently the gold standard treatment for the management of elbow PJI; there are no clear management guidelines or consensus as to which revision strategy is the most effective because of the very limited data available. Other treatment options for infected elbow prostheses have been reported to be associated with acceptable outcomes and these include DAIR and resection arthroplasty [7, 28]. However, debridement with suppressive antibiotic therapy has been reported to offer benefit only in the early post-operative period [9]. Resection arthroplasty is considered to be a salvage procedure which is only of benefit for frail patients and those in whom elbow function is not a major concern $[7,12]$. The two-stage revision strategy is commonly associated with high infection control rates in lower limb replacement [14, 15] and shoulder arthroplasty [16], but it requires two major surgical procedures which usually cause substantial functional impairment and prolonged periods of hospitalisation [29]. It is also associated with high health service costs [8]. Data, generally from hip and knee joints, suggest that the one-stage revision strategy may have several advantages over the two-stage revision which include shorter periods of hospitalisation and antibiotic therapy, better functional results, and significant cost savings [30, 31]. Currently, 
there is inadequate evidence to show that these advantages may be applicable to elbow joint PJI. Given the significant burden of elbow PJI to the patient, the surgeon and society as a whole, there is a great need for further research in this area to address the existing gaps. We encourage investigators with access to case series on elbow joint PJI treatment to publish their outcomes on follow-up of their patients.

Given the sparse evidence on the topic, our review represents the first attempt at bringing all the evidence together using a systematic approach. The search strategy was comprehensive and involved multiple databases, with manual reference scanning and no language restrictions; which made it unlikely that we had missed any relevant study conducted on the topic. Though the data was limited and sparse on outcome measures, harmonisation to consistent comparisons enabled interpretation of the findings. We took into account the low event rates reported by the majority of the studies. Finally, we conducted a detailed assessment of the methodological quality of the included studies based on a well validated tool. There were important limitations to this review and these were all related to the included studies. The included studies recruited participants between 1978 to 2016; hence given that some of these studies were conducted several decades ago, inclusion of these data may not reflect current standards of practice, as TER implant designs have changed over time [32]. Prosthetic designs and surgical techniques have improved as well as the introduction of newer and more effective antimicrobial therapies, therefore including these older studies could have biased the outcomes. There was a small possibility that two of the two-stage revision studies had overlapping patients [13, 24] and attempts to get the original authors to confirm or refute this proved futile. Whereas some studies did not report the definition of PJI, those reported by other studies varied and these could have biased the findings; however, the majority of studies diagnosed PJI using similar criteria. A robust comparison of all outcomes of interest could not be made between the two revision strategies because of the limited number of published studies and outcome data reported. The sample sizes were small and had very low event rates. These limitations precluded detailed analyses and effective comparisons. The findings therefore need to be interpreted with caution. However, the current findings are timely and relevant because they provide substantial insight on the huge gaps in the existing literature. In the absence of case series to compare the effectiveness of the two revision strategies, there is a potential that data from national joint registries may be useful in answering these questions.

\section{Conclusions}

No strong conclusions can be drawn because of limited data. The one-stage revision strategy may be potentially as clinically effective as the two-stage revision for the treatment of elbow PJI, but further data is required. Remarkable findings are the clear gaps in the existing literature and studies are urgently warranted to evaluate the clinical effectiveness of one- and two-stage revision strategies for treating elbow PJI.

\section{Supplementary information}

Supplementary information accompanies this paper at https://doi.org/10. 1186/s12891-019-2848-X.

Additional file 1: Table S1. Literature search strategy.

Additional file 2: Figure S1. Rates of non-infection related adverse events in infected elbow prostheses treated by one- and two-stage revision.

\section{Abbreviations}

Cl: Confidence interval; DAIR: Debridement, treatment with antibiotics and retention of the prosthesis; MEPS: Mayo elbow performance score; MINORS: Methodological Index for Non-Randomised Studies; PJl: Prosthetic joint infection; RCT: Randomised controlled trial; TER: Total elbow replacement

\section{Acknowledgements}

Not applicable.

\begin{abstract}
Authors' contributions
SKK, ADB, MRW, and AWB conceived and designed the study. SKK and ADB acquired data. SKK analysed and interpreted the data. SKK drafted the manuscript. SKK, ADB, MRW, and AWB critically revised the manuscript for important intellectual content. SKK had full access to all of the data in the study and takes responsibility for the manuscript content. All authors have read and approved the final manuscript.
\end{abstract}

\section{Funding}

This article presents independent research funded by the National Institute for Health Research (NIHR) under its Programme Grants for Applied Research program (RP-PG-1210-12005). This study was supported by the NIHR Biomedical Research Centre at University Hospitals Bristol NHS Foundation Trust and the University of Bristol (BRC-1215-20011). The views expressed in this publication are those of the authors and not necessarily those of the $\mathrm{NIHR}$ or the Department of Health and Social Care. These sources had no role in design and conduct of the study; collection, management, analysis, and interpretation of the data; and preparation, review, or approval of the manuscript.

\section{Availability of data and materials}

The sources of the data analysed in the study have been detailed in the manuscript.

Ethics approval and consent to participate Not applicable.

Consent for publication

Not applicable.

Competing interests

The authors declare that they have no competing interests. 
Received: 15 July 2019 Accepted: 23 September 2019

Published online: 22 October 2019

\section{References}

1. Krenek L, Farng E, Zingmond D, SooHoo NF. Complication and revision rates following total elbow arthroplasty. J Hand Surg Am. 2011;36(1):68-73.

2. National Joint Registry for England and Wales: 15th Annual Report. 2018. Accessed from http://www.njrreports.org.uk/Portals/O/PDFdownloads/NJR\%2 015th\%20Annual\%20Report\%202018.pdf.

3. Little CP, Graham AJ, Carr AJ. Total elbow arthroplasty: a systematic review of the literature in the English language until the end of 2003. J Bone Joint Surg Br. 2005;87(4):437-44.

4. Morrey BF, Bryan RS. Infection after total elbow arthroplasty. J Bone Joint Surg Am. 1983;65(3):330-8.

5. Park SE, Kim JY, Cho SW, Rhee SK, Kwon SY. Complications and revision rate compared by type of total elbow arthroplasty. J Shoulder Elb Surg. 2013; 22(8):1121-7.

6. Kasten MD, Skinner HB. Total elbow arthroplasty. An 18-year experience. Clin Orthop Relat Res. 1993;290:177-88.

7. Rhee YG, Cho NS, Park JG, Song JH. Resection arthroplasty for periprosthetic infection after total elbow arthroplasty. J Shoulder Elb Surg. 2016;25(1):105-11.

8. Wagner ER, Ransom JE, Kremers HM, Morrey M, Sanchez-Sotelo J. Comparison of the hospital costs for two-stage reimplantation for deep infection, single-stage revision and primary total elbow arthroplasty. Shoulder Elbow. 2017;9(4):279-84.

9. Wolfe SW, Figgie MP, Inglis AE, Bohn WW, Ranawat CS. Management of infection about total elbow prostheses. J Bone Joint Surg Am. 1990;72(2): 198-212.

10. Somerson JS, Morrey ME, Sanchez-Sotelo J, Morrey BF. Diagnosis and Management of Periprosthetic Elbow Infection. J Bone Joint Surg Am. 2015; 97(23):1962-71.

11. Zarkadas PC, Cass B, Throckmorton T, Adams R, Sanchez-Sotelo J, Morrey BF. Long-term outcome of resection arthroplasty for the failed total elbow arthroplasty. J Bone Joint Surg Am. 2010;92(15):2576-82.

12. Yamaguchi $\mathrm{K}$, Adams RA, Morrey BF. Infection after total elbow arthroplasty. J Bone Joint Surg Am. 1998;80(4):481-91.

13. Achermann Y, Vogt M, Spormann C, Kolling C, Remschmidt C, Wust J, Simmen B, Trampuz A. Characteristics and outcome of 27 elbow periprosthetic joint infections: results from a 14-year cohort study of 358 elbow prostheses. Clin Microbiol Infect. 2011;17(3):432-8.

14. Kunutsor SK, Whitehouse MR, Blom AW, Beswick AD. Inform team: reinfection outcomes following one- and two-stage surgical revision of infected hip prosthesis: a systematic review and meta-analysis. PLoS One. 2015;10(9):e0139166.

15. Kunutsor SK, Whitehouse MR, Lenguerrand E, Blom AW, Beswick AD. Inform team: re-infection outcomes following one- and two-stage surgical revision of infected knee prosthesis: a systematic review and meta-analysis. PLoS One. 2016:11(3):e0151537.

16. Kunutsor SK, Wylde V, Beswick AD, Whitehouse MR, Blom AW. One- and two-stage surgical revision of infected shoulder prostheses following arthroplasty surgery: a systematic review and meta-analysis. Sci Rep. 2019; $9(1): 232$.

17. Kunutsor SK, Whitehouse MR, Blom AW, Board T, Kay P, Wroblewski BM, Zeller V, Chen SY, Hsieh PH, Masri BA, et al. One- and two-stage surgical revision of peri-prosthetic joint infection of the hip: a pooled individual participant data analysis of 44 cohort studies. Eur J Epidemiol. 2018;33(10): 933-46.

18. Stroup DF, Berlin JA, Morton SC, Olkin I, Williamson GD, Rennie D, Moher D, Becker BJ, Sipe TA, Thacker SB, et al. Meta-analysis of observational studies in epidemiology. JAMA. 2000;283(15):2008-12.

19. Moher D, Liberati A, Tetzlaff J, Altman DG. Preferred reporting items for systematic reviews and meta-analyses: the PRISMA statement. PLoS Med 2009;6(7):e1000097.

20. Slim K, Nini E, Forestier D, Kwiatkowski F, Panis Y, Chipponi J. Methodological index for non-randomized studies (MINORS): development and validation of a new instrument. ANZ J Surg. 2003;73(9):712-6.

21. Freeman MF, Tukey JW. Transformations related to the angular and the square root. Ann Math Statist. 1950;21:607-11.

22. Yamaguchi K, Adams RA, Morrey BF. Semiconstrained total elbow arthroplasty in the context of treated previous infection. J Shoulder Elb Surg. 1999;8(5):461-5.
23. Gille J, Ince A, Gonzalez O, Katzer A, Loehr JF. Single-stage revision of periprosthetic infection following total elbow replacement. J Bone Joint Surg Br. 2006;88(10):1341-6.

24. Spormann C, Achermann Y, Simmen BR, Schwyzer HK, Vogt M, Goldhahn J, Kolling C. Treatment strategies for periprosthetic infections after primary elbow arthroplasty. J Shoulder Elb Surg. 2012;21(8):992-1000.

25. Peach CA, Nicoletti S, Lawrence TM, Stanley D. Two-stage revision for the treatment of the infected total elbow arthroplasty. Bone Joint J. 2013;95B(12):1681-6.

26. Rudge WBJ, Eseonu K, Brown M, Warren S, Majed A, Bayley IL, Lambert SM, Higgs D, Falworth M. The management of infected elbow arthroplasty by two-stage revision. J Shoulder Elb Surg. 2018;27(5):879-86.

27. Zmistowski B, Pourjafari A, Padegimas EM, Sheth M, Cox RM, Ramsey ML, Horneff JG 3rd, Namdari S. Treatment of periprosthetic joint infection of the elbow: 15-year experience at a single institution. J Shoulder Elb Surg. 2018; 27(9):1636-41.

28. Kunutsor SK, Beswick AD, Whitehouse MR, Wylde V, Blom AW. Debridement, antibiotics and implant retention for periprosthetic joint infections: a systematic review and meta-analysis of treatment outcomes. J Infect. 2018; 77(6):479-88

29. Matthews PC, Berendt AR, MCNally MA, Byren I. Diagnosis and management of prosthetic joint infection. BMJ. 2009:338:b1773.

30. Parkinson RW, Kay PR, Rawal A. A case for one-stage revision in infected total knee arthroplasty? Knee. 2011;18(1):1-4.

31. Kendoff D, Gehrke T. Surgical management of periprosthetic joint infection: one-stage exchange. J Knee Surg. 2014;27(4):273-8.

32. Geurts EJ, Viveen J, van Riet RP, Kodde IF, Eygendaal D. Outcomes after revision total elbow arthroplasty: a systematic review. J Shoulder Elb Surg. 2019;28(2):381-6.

\section{Publisher's Note}

Springer Nature remains neutral with regard to jurisdictional claims in published maps and institutional affiliations.
Ready to submit your research? Choose BMC and benefit from:

- fast, convenient online submission

- thorough peer review by experienced researchers in your field

- rapid publication on acceptance

- support for research data, including large and complex data types

- gold Open Access which fosters wider collaboration and increased citations

- maximum visibility for your research: over $100 \mathrm{M}$ website views per year

At BMC, research is always in progress.

Learn more biomedcentral.com/submissions 